\title{
BMJ Open Outcomes of HIV treatment from the private sector in low-income and middle-income countries: a systematic review protocol
}

\author{
Gitau Mburu (D) , ${ }^{1,2}$ Ewemade Igbinedion, ${ }^{3}$ Sin How Lim, ${ }^{4}$ Aung Zayar Paing, ${ }^{5}$ \\ Siyan Yi, ${ }^{6,7,8}$ Stefan Elbe, ${ }^{1}$ Grace W Mwai ${ }^{9}$
}

To cite: Mburu G, Igbinedion E, Lim SH, et al. Outcomes of HIV treatment from the private sector in lowincome and middle-income countries: a systematic review protocol. BMJ Open 2020;10:e031844. doi:10.1136/ bmjopen-2019-031844

- Prepublication history and additional material for this paper are available online. To view these files, please visit the journal online (http://dx.doi org/10.1136/bmjopen-2019031844).

Received 21 May 2019 Revised 04 December 2019 Accepted 18 December 2019

Check for updates

(C) Author(s) (or their employer(s)) 2020. Re-use permitted under CC BY-NC. No commercial re-use. See rights and permissions. Published by BMJ.

For numbered affiliations see end of article.

Correspondence to

Dr Gitau Mburu;

g.mburu@sussex.ac.uk

\section{ABSTRACT}

Introduction Private sector provision of HIV treatment is increasing in low-income and middleincome countries (LMIC). However, there is limited documentation of its outcomes. This protocol reports a proposed systematic review that will synthesise clinical outcomes of private sector HIV treatment in LMIC.

Methods and analysis This review will be conducted in accordance with the preferred reporting items for systematic review and meta-analyses protocols. Primary outcomes will include: (1) proportion of eligible patients initiating antiretroviral therapy (ART); (2) proportion of those on ART with $<1000$ copies $/ \mathrm{mL}$; (3) rate of all-cause mortality among ART recipients. Secondary outcomes will include: (1) proportion receiving Pneumocystis jiroveci pneumonia prophylaxis; (2) proportion with $>90 \%$ ART adherence (based on any measure reported); (3) proportion screened for non-communicable diseases (specifically cervical cancer, diabetes, hypertension and mental ill health); (iv) proportion screened for tuberculosis. A search of five electronic bibliographical databases (Embase, Medline, PsychINFO, Web of Science and CINAHL) and reference lists of included articles will be conducted to identify relevant articles reporting HIV clinical outcomes. Searches will be limited to LMIC. No age, publication date, study-design or language limits will be applied. Authors of relevant studies will be contacted for clarification. Two reviewers will independently screen citations and abstracts, identify full text articles for inclusion, extract data and appraise the quality and bias of included studies. Outcome data will be pooled to generate aggregative proportions of primary and secondary outcomes. Descriptive statistics and a narrative synthesis will be presented. Heterogeneity and sensitivity assessments will be conducted to aid interpretation of results.

Ethics and dissemination The results of this review will be disseminated through a peer-reviewed scientific manuscript and at international scientific conferences. Results will inform quality improvement strategies, replication of identified good practices, potential policy changes, and future research.

PROSPERO registration number CRD42016040053.
Strengths and limitations of this study

- This study will provide synthesised information related to outcomes of private sector provision of HIV treatment in low-income and middle-income countries (LMIC).

- This protocol adheres to Preferred Reporting Items for Systematic Review and Meta-Analysis Protocols guidelines to ensure objectivity in literature search, synthesis and reporting of outcomes from five databases.

- The results of this review will inform policy, practice and future research related to private sector provision of HIV treatment in LMIC.

- Anticipated potential limitations include high degree of heterogeneity in outcome measures (such as viral suppression) and settings.

- Exclusion of grey literature may prevent useful practical information and data being considered.

\section{INTRODUCTION}

The HIV epidemic has placed a disproportionate burden on health systems in low-income and middle-income countries (LMIC). ${ }^{1}$ As antiretroviral therapy (ART) is being scaled up, the infrastructure, information systems, diagnostics and human resources required for the provision and sustained monitoring of HIV treatment are becoming increasingly inadequate in countries with high prevalence and incidence of $\mathrm{HIV}^{2-6}$ Currently, over 21.7 million people in LMIC are on $\mathrm{ART}^{7}$ having risen from 1 million in 2001. ${ }^{8}$ The number of people living with HIV who are immediately eligible for treatment has increased significantly following WHO guidelines for early treatment regardless of CD4 count. ${ }^{9}$

Rising numbers of people in need of HIV treatment has placed significant challenges and burden on health systems in LMIC. In response to the increasing burden on public health facilities, new and innovative models 
of providing ART have emerged. These include the deployment of community health workers to support community-based ART distribution, ${ }^{10}$ task-shifting of ART provision to nurses instead of doctors ${ }^{11}$ spacing of patient appointments to reduce overcrowding at clinics and decentralisation of ART from congested tertiary facilities to local primary health centres. ${ }^{12}$ Of particular importance, however, is the proliferation of private sector clinics that are playing an increasing, but relatively nascent role in the provision of ART. ${ }^{13}$ As a result of this expansion, there is an evolving realisation that the private sector could contribute to ART provision, alleviating pressure on public facilities. ${ }^{13}$

However, there has been uncertainty regarding the quality of ART services offered through new models of treatment provision. ${ }^{14}$ Although exploration of alternative ART delivery models is a necessity, short-term and long-term patient outcomes should not be compromised. ${ }^{1}$ Evidence from several countries suggests that enforcement of policies and dissemination of updated clinical guidelines in private clinics is generally more difficult compared with public facilities, ${ }^{15}{ }^{16}$ which may affect quality. User fees borne by clients accessing services from private practitioners could also affect quality for example, by contributing to missed appointments when patients lack fees. ${ }^{13}$ These concerns are amplified by an ongoing ideological dilemma regarding the extent to which the private sector should be leveraged to provide ART, when the primary responsibility of providing universal healthcare rests with governments. ${ }^{17} 18$ A large number of people in countries such as South Africa, India, Namibia, Papua New Guinea and Tanzania are accessing ART through private providers. ${ }^{19-23}$ Yet searches of Embase, Cochrane Library and PubMed databases suggest that there is limited literature describing pooled outcomes of ART delivered by the private sector in LMIC.

In response to this gap, this protocol documents a proposed systematic review, which will describe and synthesise the outcomes of private sector provision of ART in LMIC. The specific research question is: what are the clinical outcomes of ART from private sector in LMIC? This review will not compare private versus public provision of ART. The first objective will be to aggregate clinical outcomes of ART provision by the private sector in LMIC. The second objective will be to identify evidence gaps, if any. Depending on the findings, recommendations for policy, practice and future research will be made.

\section{METHODS}

This review will be conducted in accordance with the Preferred Reporting Items for Systematic Review and Meta-Analysis Protocols (PRISMA-P) 2015 statement, ${ }^{24} 25$ and this protocol follows the PRISMA-P statement and checklist $^{24}$ (see online supplementary appendix 1 for the checklist).

\section{Data sources}

A search will be conducted to identify published peerreviewed literature in the following databases: Embase, Medline, PsychINFO, Web of Science and CINAHL. Reference lists of included papers will be manually searched for additional relevant citations. Additionally, 'cited by' tool in Google Scholar will be used to identify potentially relevant studies. Non-peer reviewed literature will not be included. Authors of ongoing studies and in-press literature will be contacted for information regarding additional studies or missing data.

\section{Search strategy}

A search will be conducted by two health practitioners (GM and EI), who are familiar with HIV service provision and systematic searches, using a search strategy developed for searching electronic sources (see online supplementary appendix 2). This search strategy will use a combination of relevant keywords and geographical limits, the latter to ensure that search focusses on LMIC. No age, publication date or language limits will be applied. This search strategy will be further adapted and tailored for use with each database, using Boolean operators, truncations, proximity operators and Medical Subject Heading, as appropriate for each database.

\section{Inclusion and exclusion criteria \\ Study design}

All study designs, including primary non-randomised and randomised controlled trials (RCTs), as well as primary observational studies, such as before and after, cohort, case-control and cross-sectional studies, will be included. Studies will be excluded if they are not reporting primary data, such as non-original research, secondary reports, commentaries, editorials and reviews. Qualitative, economic analysis and mathematical modelling studies will be excluded, primarily because the interest in this review is clinical in nature as shown in table 1.

\section{Domain}

Studies will be included if they are related to HIV.

\section{Population}

Studies will be included if they report data generated from HIV-infected participants, regardless of age, gender and sex.

\section{Intervention}

The review will include studies in which participants are exposed to private-sector provision of ART, regardless of duration of exposure. For the purpose of this review, the definition of private sector is hinged on the setting in which treatment is provided, such as private clinics, mission hospitals and facilities, for a fee to the practitioners. Studies conducted in public health facilities under ministries of health, will be excluded, even if non-governmental entities may have been involved in providing technical assistance. Because or definition of private sector is hinged on the payment of a fee 
Table 1 Primary and secondary outcomes of the review

\begin{tabular}{ll}
\hline Outcome level & Outcomes \\
\hline Primary outcomes & $\begin{array}{l}\text { Proportion of eligible patients initiating ART } \\
\text { Proportion of those on ART with }<1000 \mathrm{copies} / \mathrm{mL} \\
\text { Rate of all-cause mortality }\end{array}$ \\
$\begin{array}{l}\text { Secondary outcomes } \\
\text { Proportion receiving Pneumocystis jiroveci pneumonia prophylaxis } \\
\text { Proportion with }>90 \% \text { ART adherence (based on any measure reported) } \\
\text { Proportion screened for non-communicable diseases (that is cervical cancer, diabetes, hypertension } \\
\text { and mental health) } \\
\text { Proportion screened for tuberculosis }\end{array}$ \\
\hline
\end{tabular}

ART, antiretroviral therapy.

to private sector, it is possible that fee payment could be made by external entities-such as governments, on behalf of patients. Studies that report outcomes from such outsourced contracting of private sector for a fee by governments will be included. Studies that include both private and public sector will be included, but only data related to private sector patients will be extracted. Studies that report outcomes wholly from public sector, and those that fail to provide an adequate description of whether the setting was a private or public facility will be excluded. In addition, studies that include both private and public sector but fail to segregate data from these two settings will be excluded.

\section{Comparator}

This review does not focus on comparison, but rather, pooling of outcomes from the private sector. Studies will be included regardless of whether they report comparative outcomes from a control arms.

\section{Outcomes}

Relevant patient-related primary outcomes are: initiation of ART, retention, viral suppression, all-cause mortality and adherence to ART.

Secondary outcomes will include indicators of quality of care related to opportunistic infections and noncommunicable diseases. The denominators of these proportional primary and secondary outcomes will be the total number of patients under the care of private practitioners within included studies. Studies that do not report on any of these primary or secondary outcomes, or those that fail to provide an adequate description of the outcomes will be excluded (table 1).

\section{Geographical context}

Studies will be included if they are conducted in LMIC, as defined by the World Bank (http:/ / data.worldbank.org/ country). Studies will be excluded if they report research that wholly took place in developed countries. In the event that studies report multicountry research, relevant data from eligible LMIC will be considered, if adequately disaggregated by country.

\section{Study selection and data extraction}

All citations will be imported to EndNote and duplicates removed prior to screening. Studies will be independently screened by two review team members (GM, EI) in two stages. Reviewers will not be blinded to journal or author details. In the first stage, titles and abstracts will be screened to exclude ineligible studies, using a broad, customised and pilot tested checklist for study selection (see online supplementary appendix 3). In the second stage, full text versions of selected abstracts will be downloaded/retrieved and assessed independently by the two reviewers to ensure that inclusion criteria are met. Screening and selection of studies will be facilitated by the creation of appropriately labelled subfolders in EndNote, to segregate studies for inclusion and exclusion.

At each stage, selected papers will be compared between the two reviewers for concordance. In the event of uncertainty or disagreement, the two authors will confer and discuss with each other and, if necessary, a third review author (GWM) to reach consensus. Where separate papers report data from the same cohort, these data will be reported as one study and only data from the most recent paper with the longest duration of follow-up will be extracted to limit bias arising from multiple reporting of data from same participants. ${ }^{26}{ }^{27}$ When abstracts and subsequent included papers are not available in English, translators will be sought. In accordance with PRISMA-P guidelines for a systematic review, ${ }^{24}$ a flow diagram illustrating the literature search, article selection and final included studies will be generated.

\section{Data extraction}

A standard data extraction form will be constructed for this review in Microsoft Word and piloted on at least three random studies selected from an initial list of included studies. Based on the pilot, the data extraction form will be modified as necessary. Data will be extracted by two authors working independently (GM and EI). Once all data are extracted, a 'Characteristics of Included Studies' table will be created using methods suggested by the Cochrane Handbook for Systematic Reviews of Interventions to summarise the included studies. ${ }^{28}$ Extracted information will include the first author, year of publication, 


\begin{tabular}{|c|c|}
\hline Field & Description \\
\hline Author & $\begin{array}{l}\text { First author } \\
\text { Multiple papers reporting on the same participants are treated as one study }\end{array}$ \\
\hline Year & Year the study was published \\
\hline Country of study & $\begin{array}{l}\text { Country in which the study was conducted } \\
\text { For multicountry studies, only data related to low-income or middle-income countries will be } \\
\text { extracted }\end{array}$ \\
\hline Design & Description of study design and research methodology \\
\hline Setting & Site of study (community, health facility, etc), patient recruitment and conduct of the study \\
\hline Study population & Study population characteristics (age, sex, sexual identity or other characteristics) \\
\hline Intervention & $\begin{array}{l}\text { Description of ART-related clinical services provided by healthcare providers in different } \\
\text { countries including timing and duration }\end{array}$ \\
\hline Primary outcomes & $\begin{array}{l}\text { Summary of primary outcomes assessed } \\
\text { Where reported, proportions, rates or other measures of risks, as well as HR and confidence } \\
\text { limits will be included } \\
\text { Calculated outcomes will be marked as 'calculated' }\end{array}$ \\
\hline Secondary outcomes & $\begin{array}{l}\text { Summary of secondary outcomes assessed } \\
\text { Where reported, proportions, rates or other measures of risks, as well as HR and confidence } \\
\text { limits will be included } \\
\text { Calculated outcomes will be marked as 'calculated' }\end{array}$ \\
\hline Quality assessment & Quality scores of included studies \\
\hline Limitations & Sources of bias \\
\hline
\end{tabular}

ART, antiretroviral therapy; HR, hazard ratio.

study design, research methodology, country in which the study was conducted, participants' characteristics and recruitment, intervention type and duration, primary and secondary outcomes with point estimates and confidence limits, and study limitations (table 2).

Extracted data will be compared between the two reviewers for each included study. Discrepancies will be documented and resolved via review of the full text of manuscripts, discussion and consensus between the two reviewers. Data assumed to be missing at random may not be important and will be ignored, however, one attempt will be made to contact, by email, the corresponding authors of studies containing data not assumed to be missing at random. If the authors provide no additional information, the two reviewers will make a decision as to whether to include the study in the final review. Once all data are extracted, a table will be used to provide a summary overview of the included articles' characteristics.

\section{Quality and bias assessment}

Two review authors (GM, EI) will evaluate the risk of different types of biases, including selection bias, intervention performance bias, attrition bias, detection bias and reporting bias using the Cochrane Collaboration's tool for assessing risk of bias. ${ }^{28}$ In addition, assessment of methodological quality will be conducted using NewcastleOttawa Quality Assessment Scale, which is a validated tool for assessing quantitative cross sectional, case-control and cohort studies. ${ }^{29}$ Scores between 7 and the maximum score of 9 will be defined as high quality, scores between
4 and 6 will be defined as intermediate quality and scores between 1 and 3 will be defined as low quality. We do not anticipate to find eligible RCTs, and as stated earlier in this protocol, qualitative studies will be excluded. Two authors, GM and EI will conduct risk and quality assessments and will score each study independently.Discrepancies in scoring will be resolved by discussion with a third author (GWM). Studies will be included regardless of risk of bias and quality scores, but sensitivity analysis will be conducted to ascertain the impact of their inclusion.

\section{Data synthesis and presentation}

All available quantitative data will be pooled for each outcome using STATA version 16, SPSS version 23 or RevMan. For dichotomous outcomes, we will analyse and pool and aggregate data related to proportions of having the outcome of interest and 95\% CIs using a random effect model. For continuous outcomes, we will pool data based on the arithmetic means (or medians). To aggregate quantitative outcomes, meta-analysis will be conducted if at least five studies providing data regarding any primary outcome are identified for inclusion, unless the data are substantially heterogeneous statistically. If there is insufficient data for aggregative pooling, that is, less than five studies reporting same primary outcomes, the results will be presented in a narrative format. However, a final decision will be made once available data have been obtained and systematically appraised.

Some heterogeneity is anticipated and could be related to the intervention models, clinic settings, outcome 
measures, types of patients and reported effects. To assess if variability in outcomes will be due to chance or true heterogeneity, $\chi^{2}$ test for heterogeneity will be conducted for each outcome, and the results quantified using $\mathrm{I}^{2}$ statistic. An $\mathrm{I}^{2}$ value greater than $50 \%$ will be considered an indicator of substantial heterogeneity. ${ }^{28}$ In these cases, pooled results from the meta-analysis will not be reported; rather, a narrative approach will be utilised to report the findings. If sufficient (at least five, homogeneous) studies are identified, subgroup analysis will be conducted based on age (adult vs children $<10$ years old) and sex.

The authors will make one attempt to contact, by email, the corresponding authors of studies with missing data, unless such data are assumed to be of limited impact on the findings. If corresponding authors provide no additional information within 4 weeks, GM and EI will determine whether to include the study in the final review. Where possible, we will compute point estimates, such as risk ratios, $95 \%$ CIs and $p$ values, based on intention-totreat analysis. Assumptions (eg, those related to computed values) will be reported. Using sensitivity analyses, effects of any arbitrary decisions and calculated values on pooled proportions will be assessed by comparing the estimated proportions with and without these data.

Before carefully deriving conclusions and recommendations, the overall limitations of the review itself will be considered. Given the high volume of synthesised information, supplementary materials will be provided online in formats suitable for abstracts, manuscripts and PowerPoint presentations.

\section{Protocol registration and deviations}

This protocol is registered in PROSPERO (http://www. crd.york.ac.uk/PROSPERO/) to prevent duplication, limit reporting bias and strengthen transparency. ${ }^{30}$ The review commenced in 2019 is expected to be completed in 2020. To further limit reporting bias, and strengthen transparency, deviations from this protocol will be disclosed and discussed in the final published review.

\section{Ethics and dissemination}

The results of this review will be disseminated through publication in a peer-reviewed scientific manuscript and at international scientific conferences. Short summaries of the review will be disseminated through mass emails and social media, as these methods are often effective. ${ }^{3132}$ Workshop and symposium presentations will be utilised selectively based on audience. ${ }^{33}$

\section{Patient and public involvement}

Although involvement of patients is an important ethical element of research, patients were not involved in the development of this systematic review protocol.

\section{DISCUSSION}

Faced with overburdened public health systems, there is an increasing interest in the role that private providers could play in the delivery of ART in LMIC. Evidence suggests that a large number of people in countries such as South Africa, India, Namibia, Papua New Guinea and Tanzania are accessing ART through private providers. ${ }^{19-23}$ However, the concept of private providers in healthcare is not new, nor is it confined to ART. The role of the private sector in the provision of immunisation, family planning and other primary healthcare services in LMIC has been a focus of past and contemporary literature, ${ }^{34} 35$ and there is growing consensus that private healthcare systems have unique advantages over public health sectors, such as convenience. ${ }^{1835}$ However, there is conflicting evidence regarding the extent to which private practitioners provide acceptable quality of care, are efficient, and adhere to clinical guidelines. ${ }^{17} 1836$ Although these concerns and contentions are neither new nor restricted to HIV services, they are particularly significant in the context of HIV, due to the large scale and lifelong nature of ART, hence the need for a systematic review. Given that private sector in LMIC is often poorly regulated compared with public sector, ${ }^{15} 16$ understanding outcomes of ART care is an essential first step towards improvement of quality. If outcomes from this review are markedly poor for example, such results would create an impetus for strengthening supervision and enforcement of quality assurance policies in the private sector.

\section{Limitations}

Overall, it is anticipated that the review will contain a high degree of heterogeneity. Thresholds for some outcomes, such as viral suppression may be defined differently in different studies. In addition, the definition of what constitutes private sector is not always straightforward and may involve subjective judgement, for example, in relation to contracted services on behalf of governments, or insurance-mediated payments. Although a thorough search strategy has been defined, it is still possible to miss some studies. Exclusion of grey literature may also prevent useful practical information and data being considered. It is possible that many private practitioners may not have reported their patient care outcomes in scientific papers. The scope of our review focusses on clinical outcomes. Although understanding the economic and other outcomes related to private sector provision of ART would be important, our study is primarily concerned with clinical results. Future reviews could explore costrelated outcomes.

\section{Implications}

Despite these possible limitations, this review will inform quality improvement strategies, and identify good practices for potential replication. It will provide a context for potential policy changes and future research. It will be useful to policy makers, public health authorities and national HIV programmes in LMIC as they seek to find alternative models of ART provision that could complement existing public health systems. 
Author affiliations

${ }^{1}$ Centre for Global Health Policy, University of Sussex, Brighton, East Sussex, UK

${ }^{2}$ Division of Health Research, Lancaster University, Lancaster, UK

${ }^{3}$ Department of Community Health, Igbinedion University Teaching Hospital, Okada,

Nigeria

${ }^{4}$ Centre of Excellence for Research in AIDS (CERiA), University of Malaya, Kuala

Lumpur, Malaysia

${ }^{5}$ Programs Department, Alliance Myanmar, Yangon, Myanmar

${ }^{6}$ Center for Global Health Research, Touro University California, Vallejo, California,

USA

${ }^{7}$ Saw Swee Hock School of Public Health, National University Singapore, Singapore,

Singapore

${ }^{8}$ Center for Population Health Research, KHANA, Phnom Penh, Cambodia

${ }^{9}$ Brighton and Sussex University Hospitals NHS Trust, Brighton, UK

Contributors GM and GWM conceived this study. GM drafted the manuscript. IE, SHL, AZP, SY, SE and GWM critically reviewed the manuscript and provided comments. All authors approved the final manuscript.

Funding The authors have not declared a specific grant for this research from any funding agency in the public, commercial or not-for-profit sectors.

Competing interests None declared.

Patient consent for publication Not required.

Provenance and peer review Not commissioned; externally peer reviewed.

Open access This is an open access article distributed in accordance with the Creative Commons Attribution Non Commercial (CC BY-NC 4.0) license, which permits others to distribute, remix, adapt, build upon this work non-commercially, and license their derivative works on different terms, provided the original work is properly cited, appropriate credit is given, any changes made indicated, and the use is non-commercial. See: http://creativecommons.org/licenses/by-nc/4.0/.

ORCID iD

Gitau Mburu http://orcid.org/0000-0002-5812-0529

\section{REFERENCES}

1 Atun R, Bataringaya J. Building a durable response to HIV/AIDS: implications for health systems. J Acquir Immune Defic Syndr 2011;57 Suppl 2:S91-5.

2 Schneider H, Blaauw D, Gilson L, et al. Health systems and access to antiretroviral drugs for HIV in southern Africa: service delivery and human resources challenges. Reprod Health Matters 2006;14:12-23.

3 Rabkin M, El-Sadr WM, De Cock KM, et al. The impact of HIV scaleup on health systems: a priority research agenda. J Acquir Immune Defic Syndr 2009;52 Suppl 1:S6-11.

4 Case A, Paxson C. The impact of the AIDS pandemic on health services in Africa: evidence from demographic and health surveys. Demography 2011;48:675-97.

5 Muhamadi L, Nsabagasani X, Tumwesigye MN, et al. Inadequate preantiretroviral care, stock-out of antiretroviral drugs and stigma: policy challenges/bottlenecks to the new WHO recommendations for earlie initiation of antiretroviral therapy (CD $<350 \mathrm{cells} / \mathrm{microL})$ in eastern Uganda. Health Policy 2010;97:187-94.

6 Mori AT, Owenya J. Stock-outs of antiretroviral drugs and coping strategies used to prevent changes in treatment regimens in Kinondoni district, Tanzania: a cross-sectional study. J Pharm Policy Pract 2014;7:3.

7 UNAIDS. Miles to go. Closing gaps breaking barriers righting injustices. global AIDS update. Geneva: UNAIDS, 2018.

8 UNAIDS. How AIDS changed everything - MDG6: 15 years, 15 lessons of hope from the AIDS response. Geneva: UNAIDS, 2015.

9 World Health Organization. WHO consolidated guidelines on the use of antiretrovirals for treating and preventing HIV infection. 2nd ed. Geneva: World Health Organization, 2016.

10 Mwai GW, Mburu G, Torpey K, et al. Role and outcomes of community health workers in HIV care in sub-Saharan Africa: a systematic review. J Int AIDS Soc 2013;16:18586.

11 Callaghan M, Ford N, Schneider H. A systematic review of taskshifting for HIV treatment and care in Africa. Hum Resour Health 2010;8:8.
12 Bemelmans M, van den Akker T, Ford N, et al. Providing universal access to antiretroviral therapy in Thyolo, Malawi through task shifting and decentralization of HIV/AIDS care. Trop Med Int Health 2010;15:1413-20.

13 Rao P, Gabre-Kidan T, Mubangizi DB, et al. Leveraging the private health sector to enhance HIV service delivery in lower-income countries. J Acquir Immune Defic Syndr 2011;57:S116-9.

14 Moore CB, Ciaraldi E. Quality of care and service expansion for HIV care and treatment. Curr HIVIAIDS Rep 2015;12:223-30.

15 Olarinoye AO, Adesina KT, Adesiyun OO, et al. Knowledge and practices of pmtct among health care providers in private hospital in Ilorin, Nigeria. Trop J Obstet Gynaecol 2014;31:39-49.

16 Chakaya J, Uplekar M, Mansoer J, et al. Public-Private mix for control of tuberculosis and TB-HIV in Nairobi, Kenya: outcomes, opportunities and obstacles. Int J Tuberc Lung Dis 2008;12:1274-8.

17 Berendes S, Heywood P, Oliver S, et al. Quality of private and public ambulatory health care in low and middle income countries: systematic review of comparative studies. PLoS Med 2011;8:e1000433.

18 Basu S, Andrews J, Kishore S, et al. Comparative performance of private and public healthcare systems in low- and middle-income countries: a systematic review. PLoS Med 2012;9:e1001244.

19 Panditrao M, Darak S, Jori V, et al. Barriers associated with the utilization of continued care among HIV-infected women who had previously enrolled in a private sector PMTCT program in Maharashtra, India. AIDS Care 2015;27:642-8.

20 Miles K, Conlon M, Stinshoff J, et al. Public-Private partnerships in the response to HIV: experience from the resource industry in Papua New Guinea. Rural Remote Health 2014;14:2868.

21 Miller JS, Mhalu A, Chalamilla G, et al. Patient satisfaction with HIV/ AIDS care at private clinics in Dar ES Salaam, Tanzania. AIDS Care 2014;26:1150-4.

22 Venter L. Firms fill antiretroviral gap in South Africa. Lancet 2005;365:1215-6.

23 Van der Veen F, Mugala-Mukungu F, Kangudi M, et al. Antiretroviral treatment in the private sector in Namibia. Int J STD AIDS 2011;22:577-80.

24 Moher D, Shamseer L, Clarke M, et al. Preferred reporting items for systematic review and meta-analysis protocols (PRISMA-P) 2015 statement. Syst Rev 2015;4:1.

25 Shamseer L, Moher D, Clarke M, et al. Preferred reporting items for systematic review and meta-analysis protocols (PRISMA-P) 2015 : elaboration and explanation. BMJ 2015;349:g7647.

26 Eliasson M. [Duplicate publication a way of embellishing research results. Unethical misuse which threatens the validity of systematic reviews and meta-analysis]. Lakartidningen 2000;97:3454-6.

27 Tramèr MR, Reynolds DJM, Moore RA, et al. Impact of covert duplicate publication on meta-analysis: a case study. BMJ 1997;315:635-40.

28 Green S, Higgins J, Alderson P, et al. Cochrane Handbook for systematic reviews of interventions. Chichester, England: WileyBlackwell, 2008.

29 Peterson J, Welch V, Losos M, et al. The Newcastle-Ottawa scale (NOS) for assessing the quality of nonrandomised studies in metaanalyses. Ottawa: Ottawa Hospital Research Institute, 2011.

30 Booth A, Clarke M, Dooley G, et al. The nuts and bolts of Prospero: an international prospective register of systematic reviews. Syst Rev 2012;1:2.

31 Murthy L, Shepperd S, Clarke MJ, et al. Interventions to improve the use of systematic reviews in decision-making by health system managers, policy makers and clinicians. Cochrane Database Syst Rev 2012;9:CD009401.

32 Oermann MH, Floyd JA, Galvin EA, et al. Brief reports for disseminating systematic reviews to nurses. Clin Nurse Spec 2006;20:233-8.

33 Wallace J, Byrne C, Clarke M. Improving the uptake of systematic reviews: a systematic review of intervention effectiveness and relevance. BMJ Open 2014;4:e005834.

34 Levin A, Kaddar M. Role of the private sector in the provision of immunization services in low- and middle-income countries. Health Policy Plan 2011;26 Suppl 1:i4-12.

35 Keesara SR, Juma PA, Harper CC. Why do women choose private over public facilities for family planning services? A qualitative study of post-partum women in an informal urban settlement in Kenya. BMC Health Serv Res 2015;15:335.

36 Tuan T, Dung VTM, Neu I, et al. Comparative quality of private and public health services in rural Vietnam. Health Policy Plan 2005;20:319-27. 